\title{
Ramsauer-Townsend effect in muonic atom scattering
}

\author{
F. Mulhauser, ${ }^{1, *}$ A. Adamczak, ${ }^{2, \dagger}$ G. A. Beer, ${ }^{3}$ V. M. Bystritsky, ${ }^{4}$ M. Filipowicz, ${ }^{5}$ M. C. Fujiwara, ${ }^{6}$ T. M. Huber, ${ }^{7}$ O. Huot, ${ }^{1}$ \\ R. Jacot-Guillarmod, ${ }^{1, \$}$ P. Kammel, ${ }^{8, *}$ S. K. Kim, ${ }^{9}$ P. E. Knowles, ${ }^{1}$ A. R. Kunselman, ${ }^{10}$ G. M. Marshall, ${ }^{6}$ A. Olin, ${ }^{6}$ \\ C. Petitjean, ${ }^{11}$ T. A. Porcelli, ${ }^{6}$ L. A. Schaller, ${ }^{1}$ V. A. Stolupin, ${ }^{4}$ J. Woźniak, ${ }^{12}$ and J. Zmeskal ${ }^{13}$ \\ (TRIUMF Muonic Hydrogen Collaboration) \\ ${ }^{1}$ Department of Physics, University of Fribourg, CH-1700 Fribourg, Switzerland \\ ${ }^{2}$ Henryk Niewodniczański Institute of Nuclear Physics, Polish Academy of Sciences, PL-31342 Cracow, Poland \\ ${ }^{3}$ Department of Physics and Astronomy, University of Victoria, Victoria, Canada, V8W $2 Y 2$ \\ ${ }^{4}$ Laboratory of Nuclear Problems, Joint Institute for Nuclear Research, Dubna 141980, Russia \\ ${ }^{5}$ Faculty of Fuels and Energy, AGH University of Science and Technology, PL-30059 Krakow, Poland \\ ${ }^{6}$ TRIUMF, Vancouver, Canada, V6T $2 A 3$ \\ ${ }^{7}$ Gustavus Adolphus College, St. Peter, Minnesota 56082, USA \\ ${ }^{8}$ University of California, Berkeley, California 94720, USA \\ ${ }^{9}$ Physics Department, Jeonbuk National University, Jeonju, Jeonbuk 561-756, S. Korea \\ ${ }^{10}$ University of Wyoming Physics, Laramie, Wyoming 82071-3905, USA \\ ${ }^{11}$ Paul Scherrer Institute, CH-5232 Villigen PSI, Switzerland \\ ${ }^{12}$ Faculty of Physics and Applied Computer Science, AGH University of Science and Technology, PL-30059 Krakow, Poland \\ ${ }^{13}$ Stefan Meyer Institut für Subatomare Physik, Austrian Academy of Sciences, A-1090 Wien, Austria
}

(Received 15 January 2006; published 30 March 2006)

We present the final results of an experimental study of $\mu d$ and $\mu t$ atom scattering in solid hydrogen cooled to $3 \mathrm{~K}$. Strong effects resulting from the Ramsauer-Townsend effect have been observed in the TRIUMF experiment E742 where muons were stopped in thin frozen layers of hydrogen. The measured RamsauerTownsend minimum energy for both $\mu d$ and $\mu t$ atoms and the minimum cross section are in agreement with theory.

DOI: $10.1103 /$ PhysRevA.73.034501

PACS number(s): 36.10.Dr, 34.50.-s, 39.10.+j

Negative muons stopping in hydrogen can form muonic hydrogen $(\mu h)$ atoms, where $h=p, d$, or $t$ depending on the hydrogen isotope. Although created in excited states, such atoms cascade to the ground state quickly $\left(10^{-9} \mathrm{~s}\right)$, where their kinetic energy is of the order of several $\mathrm{eV}$, much higher than thermal equilibrium energies. The muonic hydrogen atom is about 200 times smaller $\left(m_{\mu} / m_{e}\right.$ scaling) than the size of ordinary electronic hydrogen. The small neutral atom can easily diffuse through the surrounding medium, undergoing different types of interactions with atoms and molecules including elastic and inelastic scattering.

The scattering processes

$$
\mu d+\mathrm{H}_{2} \rightarrow \mu d+\mathrm{H}_{2}
$$

and

$$
\mu t+\mathrm{H}_{2} \rightarrow \mu t+\mathrm{H}_{2}
$$

are predicted [1-4] to show a dramatic minimum in scattering, the so-called Ramsauer-Townsend (RT) effect. This effect is due to a minimum scattering cross section $\left(\sim 10^{-21} \mathrm{~cm}^{2}\right)$ at collision energies between 2 and $30 \mathrm{eV}$.

\footnotetext{
*Present address: University of Illinois at Urbana-Champaign, Urbana, Illinois 61801, USA.

${ }^{\dagger}$ Also at Rzeszow University of Technology, PL-35959 Rzeszow, Poland.

${ }^{\ddagger}$ Present address: Nordostlich Krafwerke, Switzerland.
}

These cross sections are more than two orders of magnitude smaller than the $\mu p+\mathrm{H}_{2}$ one [5]. The result is a quasitransparency of the $\mathrm{H}_{2}$ molecules for incident $\mu d$ or $\mu t$ atoms. Theoretical calculations for muonic atoms scattering in solid or gas are performed in Refs. [4-7]. However, the improvements in those calculations are important for muonic atoms of low energy and negligible for energies above eV [5].

The development at TRIUMF of multilayer thin frozen hydrogen film targets [8-14], which produce muonic atom beams emitted into vacuum, permitted the cross sections to be probed in an interesting way. We have studied several isolated muon induced processes using a time-of-flight (TOF) method developed by the frozen target geometry [14].

The aim of the present experiment was to study and verify the cross sections in the RT minimum of $\mu d$ and $\mu t$ elastic scattering in solid hydrogen. The experiments were performed at the M20B muon channel at TRIUMF. The layout of the apparatus was shown and described in Refs. [5,15-17]. Gaseous hydrogen (or neon) was sprayed, using a special diffusion system, onto a $51 \mu \mathrm{m}$ thick gold foil, kept at $3 \mathrm{~K}$, where it froze creating thin solid films which could be maintained in high vacuum. Details of the target construction and working procedure are given in Refs. $[9,18]$.

The muons eventually stop either in the gold target support foil or in the $800 \mu \mathrm{m}$ thick solid hydrogen target where they finally form muonic atoms. The hydrogen target frozen on that foil, which is placed perpendicularly to the muon beam axis, is called the upstream target (US), and is made of pure protium or of protium with a small admixture of deuterium or tritium, depending on the experiment. 


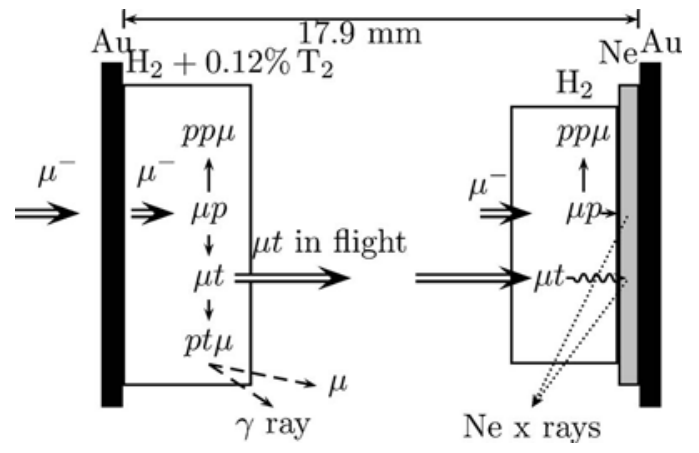

FIG. 1. Target scheme for $\mu t$. Muonic tritium atoms are emitted forward into the vacuum gap. The $p t \mu$ fusion emission is measured via the $\mathrm{NaI}(\mathrm{Tl})$ and scintillator detectors. The neon $\mathrm{x}$ rays are detected with two germanium detectors.

When deuterium or tritium was present in the US target, we used an additional downstream pure protium target (DS) frozen on a second gold foil placed parallel to the first foil but $17.9 \mathrm{~mm}$ further along the beam axis. Such an arrangement is presented in Fig. 1, where a thin layer of neon is shown sandwiched between a layer of pure protium and the DS gold foil, as used in the $\mu t$ scattering experiment. A low muon beam momentum was chosen to minimize the number of $\mu^{-}$stops in the DS protium layer [15], so that the muon stopped mainly in the US gold foil and hydrogen target layers.

Neon was used to detect the scattered muonic $\mu t$ atoms which left the DS hydrogen layer and subsequently transferred the muon to the neon. The resulting emission of $207 \mathrm{keV}$ x rays from the $2 p-1 s \mu \mathrm{Ne}$ transition was observed by two $\sim 100 \mathrm{~cm}^{3}$ germanium crystals with a time resolution of $10-12 \mathrm{~ns}$, full width at half maximum. Plastic scintillators were located around the target to detect the muon decay electrons.

The US target was composed of protium with a small concentration of $\mathrm{D}_{2}$ (or $\mathrm{T}_{2}$ ), which served as a source of energetic $\mu d(\mu t)$ atoms emitted from the layer into the adjacent vacuum with a mean energy of $3.5 \mathrm{eV}(9 \mathrm{eV}$ in the case of the $\mathrm{H}_{2} / \mathrm{T}_{2}$ mixture) as a result of the RT effect. In most cases $(70-75 \%)$ the muon was transferred from $\mu p$ to a deuteron or triton, forming $\mu d$ or $\mu t$ atoms, respectively. At formation, the muonic deuterium (tritium) atoms had a relatively high kinetic energy, about $45 \mathrm{eV}$, which they subsequently lost in elastic collisions, mainly with protium, until the energy reached the range of the RT minimum in the scattering cross section $\sigma\left(\mu d+\mathrm{H}_{2}\right)$. Then the mean distance between collisions increased and the hydrogen layer became effectively transparent for the $\mu d$ and $\mu t$ atoms which were then easily emitted from the solid into the adjacent vacuum. Such energetic emitted muonic atoms traveled through vacuum toward the downstream hydrogen layer (DS), and a fraction passed, after possible interactions in hydrogen, to the Ne layer, where it produced $\mathrm{Ne} x$ rays as a result of the muon transfer to neon and subsequent muonic neon deexcitation. Such $\mu d(\mu t)$ atoms gave a characteristic peak in the TOF spectrum at $\approx 1 \mu \mathrm{s}-\mathrm{a}$ time determined by the distance between the foils and the energy of the RT minimum within the energy collision scale of $\mu d(\mu t)$ atoms with $\mathrm{H}_{2}$ molecules.
TABLE I. Measurements performed for the RT study. DE (deuterium emission) stands for a layer of 1500 Torr $\ell\left(\mathrm{H}_{2}+0.05 \% \mathrm{D}_{2}\right)$ covered with 500 Torr $\ell \mathrm{H}_{2}$. TE (tritium emission)2000 Torr $\ell\left(\mathrm{H}_{2}+0.12 \% \mathrm{~T}_{2}\right)$. STE (small tritium emission)1000 Torr $\ell\left(\mathrm{H}_{2}+0.12 \% \mathrm{~T}_{2}\right)$. GMU—good muons: i.e., events when only one muon entered the apparatus (no pileup). Conversion factor (for hydrogen): 1 Torr $\ell$ corresponds to $3.4 \mu \mathrm{g} \mathrm{cm}^{-2}$ for $\mathrm{H}_{2}$ [19]. Beam momentum was $26.70 \mathrm{MeV} / \mathrm{c}$ and $26.25 \mathrm{MeV} / \mathrm{c}$ for the deuterium and tritium, respectively.

\begin{tabular}{ccccc}
\hline \hline Label & $\begin{array}{c}\text { US } \\
\text { Torr } \ell\end{array}$ & $\begin{array}{c}\text { DS protium } \\
\text { Torr } \ell\end{array}$ & $\begin{array}{c}\text { DS Neon } \\
\text { Torr } \ell\end{array}$ & GMU $\times 10^{6}$ \\
\hline D1-D2 & DE & & $100-50$ & 510.2 \\
D3 & DE & 300 & 50 & 521.8 \\
D4 & DE & 600 & 50 & 433.2 \\
T1-T2 & TE & & $30-50$ & 287.7 \\
T3 & TE & 350 & 50 & 405.3 \\
T4 & STE & 500 & 50 & 147.1 \\
\hline \hline
\end{tabular}

Clearly, not all muons were stopped in the US target. Those which reached the second foil created $\mu p$ 's in the DS protium. The $\mu p$ 's diffused to neon giving a contribution to the time spectrum at early times. Thus the resulting Ne time spectrum contained two relatively distinct components, one of them connected with the RT effect in $\mu d(\mu t)$ scattering and the other with the diffusion of $\mu p$ atoms in the solid hydrogen. The full study of $\mu p$ atom diffusion is given by Woźniak et al. [5].

Analyzing the time distribution of the $\mu d$ 's and $\mu t$ 's via the $2 p-1 s \mu \mathrm{Ne}$ transition gives information about the scattering cross sections. Table I summarizes the different measurements performed for the study of the RT effect. The last tritium measurement (T4) was not taken into account in previous publications $[15,20]$, due to a different emission target. The essential part of the analysis is the comparison of the experimental time distributions with the ones calculated by Monte Carlo. The Monte Carlo code FOW [21] was used in the planning stages of the experiment as well as for the analysis to simulate all physical processes occurring after muons pass through the entrance window of the apparatus. Muon stopping distributions along the beam axis in the different apparatus components, especially in the hydrogen layers of the target, have been taken from a special set of measurements [15] and from another Monte Carlo calculation [22]. They are used as an input to FOW. Details about the method have already been given by Woźniak et al. [5].

Muonic atom scattering in hydrogen was measured via the $\mathrm{x}$-ray time spectra of the $\mu \mathrm{Ne} 2 p-1 s$ transition at $207 \mathrm{keV}$. The time spectra events were selected within an energy window between 205.6 and $208.3 \mathrm{keV}$. The background time spectra underneath the $207 \mathrm{keV}$ peak were created using time spectra from two neighboring energy windows, namely a left background between 203.3 and $205.6 \mathrm{keV}$ and a right background between 208.3 and $210.2 \mathrm{keV}$.

Two different background evaluation procedures were used. In the first, the left and right spectra were added, then normalized by the energy window widths, and the resulting spectrum was subtracted from the time spectrum of the 


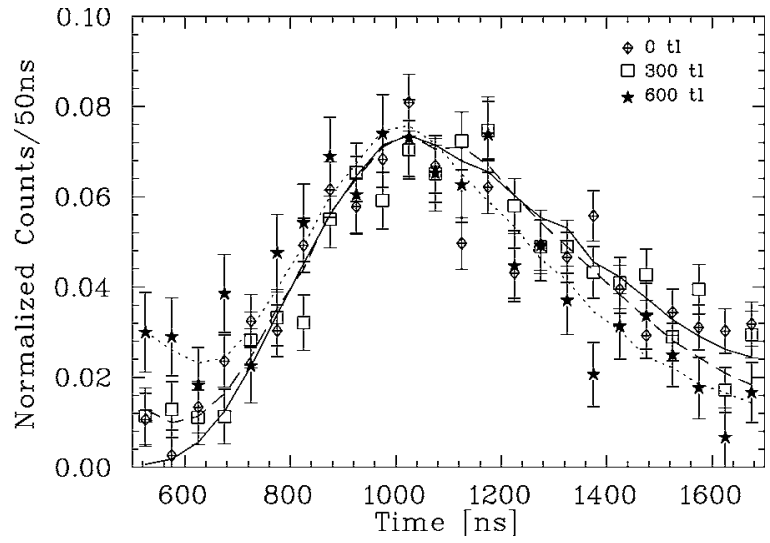

FIG. 2. Experimental TOF spectra (points with error bars) for the deuterium measurements. The solid, dashed, and dotted lines are the Monte Carlo (MC) simulations for D1-D2, D3, and D4, respectively.

$\mu \mathrm{Ne} 2 p-1 s$ peak. The second method relied on a multiparameter fit of the summed left and right backgrounds using two exponential functions (with lifetimes for muons in gold and neon), and the background predicted from the fit function was then subtracted from the $\mu \mathrm{Ne} 2 p-1 s$ peak. Since the background accounts for $70-85 \%$ of the total statistics, its removal plays an important role, especially for data at early times where there is a strong contribution from the prompt muon capture in both the neon layer and the gold foils.

Another data cleaning method resulting in a considerably better signal to background ratio was the requirement that the muon decay electron be seen after the $\mu \mathrm{Ne} 2 p-1 s$ x ray. Those electrons were detected by the scintillators during a time interval $0.2-5.2 \mu \mathrm{s}$ after the $\mu \mathrm{Ne}$ signal. This method, called the $\operatorname{del}_{e}$ condition, suppressed the background by a factor of about 300. However, useful statistics were reduced by about a factor 10. Figures 2 and 3 show background subtracted TOF spectra for the $\mu d$ and $\mu t$ atom scattering, respectively, without the del $e_{e}$ condition in all measurements. In the $\mu t$ atom scattering experiment, due to a higher RT minimum energy, a distinction between $\mu t$ and $\mu p$ processes is more difficult to perform, as shown in Fig. 16 of Woźniak et al. [5].

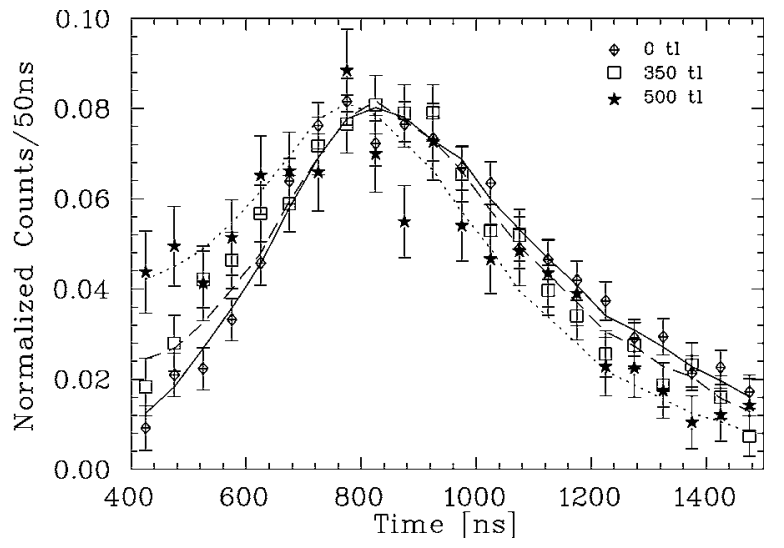

FIG. 3. Experimental TOF spectra (points with error bars) for the tritium measurements. The solid, dashed, and dotted lines are the MC simulations for T1-T2, T3, and T4, respectively.
TABLE II. Characteristic values used in the cross section parametrization for both $\mu d$ and $\mu t$ scattering. Energies are in the center of mass system (CMS) and cross sections [3] are given per one atom, i.e., for $\mu h+p \rightarrow \mu h+p$ processes.

\begin{tabular}{cccc}
\hline \hline Parameter & $\mu d$ & $\mu t$ & Units \\
\hline$E_{R}$ & 1.7 & 3.0 & $\mathrm{eV}$ \\
$E_{\min }$ & 1 & 1 & $\mathrm{meV}$ \\
$E_{\max }$ & 190.5 & 51.3 & $\mathrm{eV}$ \\
$\sigma\left(E_{R}\right)$ & 1.13 & 1.66 & $\times 10^{-21} \mathrm{~cm}^{2}$ \\
\hline \hline
\end{tabular}

A $\chi^{2}$ analysis has been performed to fit the FOW input parameters to the experimental data. The theoretical cross sections $\sigma\left(\mu d+\mathrm{H}_{2}\right)$ and $\sigma\left(\mu t+\mathrm{H}_{2}\right)$ are varied in two different ways. We determine the position of the zero of the $S$-wave amplitude and the $P$-wave cross section at the RT minimum by introducing an energy shift $\Delta E$ and a depth factor $d f$.

In contrast to previously published results [15] where the analysis consisted in varying only one parameter at a time, this final work was performed where both parameters $\Delta E$ and $d f$ were varied at the same time. The energy shift $\Delta E$ is obtained by transforming the energy axis according to $E \rightarrow E_{t r}$ and then $\sigma(E) \rightarrow \sigma\left(E_{t r}\right)$. The energy transformations are

$$
E_{t r}=\left\{\begin{array}{ll}
E+\frac{E-E_{\min }}{E_{R}-E_{\min }} \times \Delta E, & \text { for } E \leqslant E_{R} \\
E+\frac{E_{\max }-E}{E_{\max }-E_{R}} \times \Delta E, & \text { for } E>E_{R}
\end{array},\right.
$$

where $E$ is the energy value before shifting and $E_{R}$ the original value of the RT energy minimum. $E_{\min }$ and $E_{\max }$ are the minimum and maximum energy, corresponding to the left and right borders of the energy range. For $E=E_{R}$, the transformed energy $E_{t r}$ given by Eq. (2) becomes $E_{t r}=E+\Delta E$. $\sigma\left(E_{R}\right)$ is the minimum cross section for the RT energy. Thus, this parametrization can be treated as a shift and the initial parameters are found in Table II.

The depth factor $d f$ is determined via a rescaled minimum cross section without changing the position of the minimum energy $E_{R}$,

$$
\sigma_{\text {exp }}\left(E_{R}\right)=d f \times \sigma\left(E_{R}\right) .
$$

The rescaling took place in the $\mathrm{CM}$ energy range $0.5-10 \mathrm{eV}$. To preserve the smooth form of the cross sections, values within this energy range were not globally scaled by the factor $d f$, but were scaled by a factor which ranged from 1 at the borders to the factor $d f$ at the RT minimum energy.

A spline interpolation was performed between the twodimensional grid of the Monte Carlo simulation to fit the experimental data [23]. The fit starting time has been set to $t_{\min }=500(400) \mathrm{ns}$ for $\mu d(\mu t)$ atoms to avoid using the $\mu p$ diffusion part [5] of the spectra in the analysis. The fit stopping time was chosen at $t_{\max }=1700(1500)$ ns for $\mu d(\mu t)$ to avoid taking into account channels with extremely low statistics, where background subtraction plays a strong role. The 
TABLE III. Results of the two-parameter analysis for the $\mu d$ and $\mu t$ atom scattering when data are combined. Errors contain the combination of statistical and systematics uncertainties.

\begin{tabular}{ccc}
\hline \hline Label & $\begin{array}{c}\text { Depth factor } \\
d f\end{array}$ & $\begin{array}{c}\text { Energy shift } \\
\Delta E(\mathrm{eV})\end{array}$ \\
\hline D1-D4 & $1.10 \pm 0.18$ & $0.17 \pm 0.13$ \\
T1-T4 & $1.22 \pm 0.11$ & $-0.25 \pm 0.23$ \\
\hline \hline
\end{tabular}

$t_{\max }$ time was varied in the present analysis by $\pm 200 \mathrm{~ns}$, without any major effect on the fit results. Strong variations were seen in both the depth factor as well as the energy shift. Depending on the background subtraction method, the individual detector, as well as the fit interval, results scattered excessively. Variations up to $40 \%$ were obtained for both parameters. These variations were seen for both $\mu d$ and $\mu t$ measurements. As already seen in previously published works $[15,20]$, the scattered results show that the twoparameter fit is not sufficiently constrained.

Therefore, we adopted a third approach, where all $\mu d$ or $\mu t$ data were fitted together. The starting and stopping times, as well as grid size, and minimization procedures, were all similar to the previous method. The new approach, namely that for each muonic atom type the energy shift and depth factors were required to simultaneously satisfy the best fit conditon for all three DS protium thickness, provides a strong constraint of the parameters and leads to the results given in Table III. Background subtraction, individual detectors, and fit intervals are taken into account for the systematic uncertainties which are still dominant.

The agreement between experiments and theory is good for $\mu d$ and $\mu t$ atom scattering. Both theoretical minimum RT energies, $E_{R}(\mu t)=3.0 \mathrm{eV}$ and $E_{R}(\mu d)=1.7 \mathrm{eV}$, are compatible with our measurements. However, the RT determined cross section scaling factors suggest that the RT minima have a slightly shallower depth than theoretically predicted, particularly for $\mu t$ atoms.

In conclusion, we have been able to measure the Ramsauer-Townsend cross sections for $\mu d$ and $\mu t$ atom scattering with a precision of 10-20\%. More sophisticated measurements with much better statistics are needed to improve these results.

The authors would like to thank V. E. Markushin, J. L. Douglas, and M. Maier for their help during the data acquisition period and for fruitful discussions. This work is supported by the Swiss National Science Foundation, TRIUMF, the Polish Committee for Scientific Research, the Natural Sciences and Engineering Research Council of Canada, the United States National Science Foundation, the Russian Foundation for Basic Research (Grant No. 01-02-16483), and the NATO Linkage Grant No. LG9301162.
[1] M. Bubak and M. P. Faifman, JINR Preprint E4-87-464 (1987).

[2] L. Bracci et al., Muon Catal. Fusion 4, 247 (1989).

[3] C. Chiccoli et al., Muon Catal. Fusion 7, 87 (1992).

[4] A. Adamczak et al., At. Data Nucl. Data Tables 62, 255 (1996).

[5] J. Woźniak et al., Phys. Rev. A 68, 062502 (2003).

[6] A. Adamczak, Hyperfine Interact. 119, 23 (1999).

[7] A. Adamczak, Ph.D. thesis, Institute of Nuclear Physics, Kraków, Poland (2003) (unpublished).

[8] G. M. Marshall et al., Hyperfine Interact. 82, 529 (1993).

[9] P. E. Knowles et al., Nucl. Instrum. Methods Phys. Res. A 368, 604 (1996).

[10] F. Mulhauser et al., Phys. Rev. A 53, 3069 (1996).

[11] P. E. Knowles et al., Phys. Rev. A 56, 1970 (1997); 57, 3136(E) (1998).

[12] M. C. Fujiwara et al., Phys. Rev. Lett. 85, 1642 (2000).
[13] T. A. Porcelli et al., Phys. Rev. Lett. 86, 3763 (2001).

[14] G. M. Marshall et al., Hyperfine Interact. 138, 203 (2001).

[15] F. Mulhauser et al., Hyperfine Interact. 119, 35 (1999).

[16] J. Woźniak et al., Hyperfine Interact. 119, 63 (1999).

[17] V. M. Bystritsky et al., Hyperfine Interact. 138, 47 (2001).

[18] G. M. Marshall et al., Hyperfine Interact. 101/102, 47 (1996).

[19] M. C. Fujiwara et al., Nucl. Instrum. Methods Phys. Res. A 395, 159 (1997).

[20] F. Mulhauser et al., Hyperfine Interact. 138, 41 (2001).

[21] J. Woźniak, V. M. Bystritsky, R. Jacot-Guillarmod, and F. Mulhauser, Hyperfine Interact. 101/102, 573 (1996).

[22] R. Jacot-Guillarmod, Stopping Code (University of Fribourg, 1997).

[23] M. Filipowicz, V. M. Bystritsky, P. E. Knowles, F. Mulhauser, and J. Woźniak, Nucl. Instrum. Methods Phys. Res. A 547, 652 (2005). 